Institut für Lebensmittelsicherheit und -hygiene der Vetsuisse-Fakultät Universität Zürich

Direktor: Prof. Dr. Roger Stephan

Arbeit unter Leitung von PD Dr. Claudio Zweifel

Antibiotikaresistenz von Staphylococcus aureus und Koagulase-negativen Staphylokokken isoliert aus Mastitismilchproben von Schafen und Ziegen

\author{
Inaugural-Dissertation \\ zur Erlangung der Doktorwürde der \\ Vetsuisse-Fakultät Universität Zürich
}

\author{
vorgelegt von
}

\title{
Franziska Kunz
}

Tierärztin

von Ruswil, LU, Schweiz

genehmigt auf Antrag von

Prof. Dr. Roger Stephan, Referent

Prof. Dr. Michael Hässig, Korreferent

Zürich 2010 
Institut für Lebensmittelsicherheit und -hygiene der Vetsuisse-Fakultät Universität Zürich

Direktor: Prof. Dr. Roger Stephan

Arbeit unter Leitung von PD Dr. Claudio Zweifel

Antibiotikaresistenz von Staphylococcus aureus und Koagulase-negativen Staphylokokken isoliert aus Mastitismilchproben von Schafen und Ziegen

\author{
Inaugural-Dissertation \\ zur Erlangung der Doktorwürde der \\ Vetsuisse-Fakultät Universität Zürich
}

\author{
vorgelegt von
}

\title{
Franziska Kunz
}

Tierärztin

von Ruswil, LU, Schweiz

genehmigt auf Antrag von

Prof. Dr. Roger Stephan, Referent

Prof. Dr. Michael Hässig, Korreferent

Zürich 2010 


\section{INHALTSVERZEICHNIS}

1. Zusammenfassung 2

2. Einleitung 3

3. Material und Methoden 5

$\begin{array}{lr}\text { 4. Ergebnisse und Diskussion } & 7\end{array}$

$\begin{array}{ll}\text { 5. Schlussfolgerungen } & 10\end{array}$

$\begin{array}{ll}\text { 6. Literatur } & 11\end{array}$

$\begin{array}{ll}\text { 7. Tabellen } & 15\end{array}$

8. Danksagung 17 
Antibiotikaresistenz von Staphylococcus aureus und Koagulase-negativen Staphylokokken isoliert aus Mastitismilchproben von Schafen und Ziegen

F. Kunz ${ }^{1}$, S. Corti ${ }^{1}$, N. Giezendanner ${ }^{1}$, R. Stephan ${ }^{1}$, M.M. Wittenbrink ${ }^{2}$, C. Zweifel ${ }^{1}$

${ }^{1}$ Institut für Lebensmittelsicherheit und -hygiene der Universität Zürich, ${ }^{2}$ Institut für Veterinärbakteriologie der Universität Zürich

${ }^{*}$ Corresponding author. PD Dr. Claudio Zweifel, Institut für Lebensmittelsicherheit und -hygiene, Vetsuisse-Fakultät Universität Zürich, Winterthurerstrasse 272

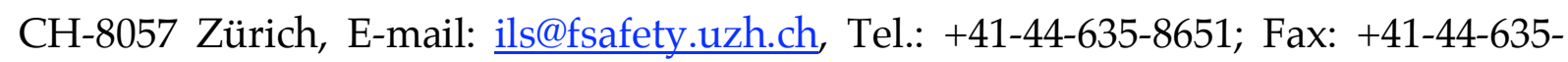
8908

Zur Publikation angenommen im Schweizer Archiv für Tierheilkunde

Als Dissertation von Franziska Kunz 


\section{Zusammenfassung}

Im Rahmen dieser Arbeit wurden aus Mastitismilchproben von Schafen und Ziegen von über 40 Betrieben der Schweiz Staphylococcus aureus und Koagulase-negative Staphylokokken (CNS) isoliert. Bei den CNS konnte mittels RestriktionsprofilAnalyse des groEL-Gens und 16S rDNA Sequenzierung gezeigt werden, dass verschiedene Spezies vorkamen, wenn auch einzelne dominierten. Die Resistenzprüfung der Mastitiserreger gegen ausgewählte Antibiotika erfolgte mittels des Mikrodilutionsverfahrens. Von den 67 S. aureus- und 208 CNS-Stämmen waren 31.3\% und $8.2 \%$ resistent gegen Penicillin, $29.9 \%$ und $1.0 \%$ resistent gegen Ampicillin, 1.5\% und 10.6\% resistent gegen Erythromycin sowie $3.0 \%$ und $7.7 \%$ resistent gegen Tetracyclin. Zudem waren 10 CNS-Stämme (4.8\%) resistent gegen Oxacillin und ein CNS-Stamm gegen Sulfamethoxazol/Trimethoprim. Die vorliegenden Daten stellen eine erste Bestandesaufnahme zur Resistenzsituation bei Mastitiserregern von Schafen und Ziegen in der Schweiz dar. Zur Mastitisbekämpfung sind aber auch flankierende und präventive Massnahmen von entscheidender Bedeutung.

Schlüsselwörter: Mastitiserreger, Kleinwiederkäuer, Antibiotikaresistenz, Minimale Hemmkonzentration (MHK) 


\section{Einleitung}

In der Schweiz werden aktuell mehr als 36'000 Milchziegen unterschiedlicher Rassen und mehr als 10'500 Milchschafe, überwiegend der Rassen Ostfriesisches Milchschaf und Lacaune, gehalten (Schweizerischer Bauernverband, www.sbv-usp.ch, Stand 14. Mai 2010). Aufgrund des Trends der Konsumenten zu möglichst naturbelassenen Produkten besteht eine steigende Nachfrage nach Schaf- und Ziegenmilch sowie daraus hergestellter Rohmilchkäse und Milchspezialitäten.

Mastitiden bakterieller Ätiologie gehören zu den häufigsten Gesundheitsproblemen von Milchkühen, -schafen und -ziegen. In der Schweiz sind im Durchschnitt zwischen 20 und 40\% der Euterhälften von Milchschafen und -ziegen von einer Mastitis betroffen (Schaeren und Maurer, 2006; Maurer und Schaeren, 2007). Akute und chronische Mastitiden stellen für Milchproduzenten ein grosses wirtschaftliches Problem dar, da sie durch Reduktion der Milchleistung, Beeinträchtigung der Milchqualität (veränderte Zusammensetzung, Beeinträchtigung der Milchqualität, Preisabzüge, zusätzlichem Arbeitsaufwand und den Behandlungskosten zu erheblichen Verlusten oder gar zum Ausmerzen von Tieren führen (Gonzalo et al., 2002; Halasa et al., 2007). Aus milchhygienischer Sicht sowie im Rahmen des Veterinary Public Health (VPH) Ansatzes sind dabei insbesondere subklinische Mastitiden von Bedeutung. Bei Kleinwiederkäuern nehmen S. aureus bei klinischen Mastitiden und Koagulase-negative Staphylokokken (CNS) bei subklinischen Mastitiden eine dominierende Stellung ein.

In der Schweiz gibt es zurzeit mit Ausnahme des Trockenstellers Nafpenzal ${ }^{\circledR}$ DC (Veterinaria, Pfäffikon, $\mathrm{CH}$ ) keine zur intramammären Anwendung bei Schafen und Ziegen zugelassene Antibiotika. Die Tierarzneimittelverordnung (TAMV, SR 812.212.27) erlaubt jedoch die Umwidmung von zur Mastitistherapie bei Kühen zugelassenen Antibiotika. Insbesondere bei Kleinwiederkäuern muss die Mastitisbekämpfung jedoch neben dem Antibiotikaeinsatz auch flankierende und präventive Massnahmen zur Ausschaltung prädisponierender Faktoren wie ungünstiger Aufstallungssysteme, fehlerhafter Betriebs- und Melkhygiene oder Fehler im Zusammenhang mit der Melkmaschine beinhalten (Bergonier et al., 2003; Zweifel et al., 2005; Contreras et al., 2007). 
Daten zur Resistenzsituation von Mastitiserregern von Schafen und Ziegen fehlten bis anhin in der Schweiz. Ziel der vorliegenden Arbeit war es (a) Mastitiserreger (S. aureus, CNS) aus Mastitismilchproben von Schafen und Ziegen zu isolieren, (b) CNS weitergehend $\mathrm{zu}$ charakterisieren respektive $\mathrm{zu}$ identifizieren und (c) Daten zur Antibiotika-Resistenzsituation der isolierten Mastitiserreger zu erheben. 


\section{Material und Methoden}

\section{Mastitismilchproben}

Im Verlauf eines Jahres wurden Milchproben von Schafen und Ziegen mit klinischer oder subklinischer Mastitis gesammelt und am Institut für Lebensmittelsicherheit und -hygiene der Universität Zürich auf Mastitiserreger untersucht. Die 275 Milchproben, in welchen $S$. aureus oder CNS nachgewiesen wurden, stammten von mehr als 40 Betrieben aus verschiedenen Regionen der Schweiz.

\section{Stammisolation, -identifizierung und-charakterisierung}

Zur Untersuchung auf Mastitiserreger wurden die Milchproben auf Blutagarplatten (Difco Laboratories, Detroit, USA; 5\% Schafblut defibriniert, Oxoid Ltd., Hampshire, UK) und auf Bromthymolblau Laktose Agarplatten (BROLAC-Agar, bioMérieux SA, Marcy-l'Etoile, F) ausgestrichen und bei $37^{\circ} \mathrm{C}$ während $24 \mathrm{~h}$ inkubiert.

Von jeder Probe mit Gram-positiven, Katalase-positiven Kokken wurde eine Kolonie-bildende Einheit mittels DNAse Test Agarplatten (Becton Dickinson AG, Allschwil, CH) auf ihre DNAse-Bildung überprüft (Fantelli und Stephan, 2003). Als Kontrollstamm diente S. aureus ATCC 25923. Zudem wurde auf Baird Parker plus Rabbit Plasma Fibrinogen Agar (Oxoid Ltd.) die Koagulasereaktion überprüft. DNAse-positive (Aufhellungszone auf DNAse Test Agar), Koagulase-positive Kolonien mit Doppelhämolyse $(\mathrm{b} / \mathrm{d})$ auf Blutagar wurden als $S$. aureus und Koagulase-negative Kolonien als CNS klassifiziert.

Die weiterführende Charakterisierung von CNS erfolgte mittels RestriktionsprofilAnalyse (PCR-RFLP) des groEL-Gens (Santos et al., 2008). Aufgrund der Restriktionsprofile wurden die CNS-Stämme verschiedenen Clustern zugeordnet. Zur Speziesidentifizierung wurde von einem oder zwei Stämmen ausgewählter PCRRFLP-Cluster ( $\geq 2$ Isolate) eine 165 rDNA Sequenzierung durchgeführt (Juretschko et al., 2002). 
Prüfung der Antibiotikaresistenz

Die Prüfung der Antibiotikaresistenz erfolgte unter Verwendung des Mikrodilutionsverfahrens (Sensititre NLV 72, Trek Diagnostik Systems Ltd., West Sussex, UK). Isolierte $S$. aureus- und CNS-Stämme wurden auf die Empfindlichkeit gegenüber Amoxicillin/Clavulansäure, Ampicillin, Cefazolin, Cephalotin, Enrofloxacin, Erythromycin, Gentamicin, Oxacillin, Penicillin G, Sulfamethoxazol/Trimethoprim und Tetracyclin getestet. Die getesteten Konzentrationsbereiche sowie die zur Beurteilung der MHK-Werte (MHK = Minimale Hemmkonzentration) verwendeten Breakpoints sind in Tabellen 1 und 2 aufgeführt. 


\section{Ergebnisse und Diskussion}

\section{Mastitisstämme}

Im Stammkollektiv der 275 Mastitisisolate von Kleinwiederkäuern wurden 67 Stämme als S. aureus und 208 als CNS identifiziert. Diese Stämme wurden jeweils zu etwa gleichen Teilen aus Milchproben von Schafen und Ziegen isoliert. Dabei stammten die 67 S. aureus-Stämme (36 von Schafen, 31 von Ziegen) aus 25 Betrieben sowie 203 der CNS-Stämme (105 von Schafen, 98 von Ziegen) aus 31 Betrieben.

\section{Charakterisierung und Identifizierung Koagulase-negativer Staphylokokken (CNS)}

CNS sind bei Schafen und Ziegen eine sehr häufige Ursache von Mastitiden, insbesondere von subklinischen (McDougall et al., 2002; Moroni et al., 2005a; Contreras et al., 2007). Für die Beurteilung der potentiellen Pathogenität bestimmter Spezies werden dabei Daten zur Charakterisierung und Speziesidentifizierung immer wichtiger (Pyörälä und Taponen, 2009; Zadoks und Watts, 2009).

Die Restriktionsprofil-Analyse einer Auswahl von 155 CNS-Stämmen ordnete diese 18 verschiedenen Gruppen (Clustern) mit unterschiedlichen Restriktionsmustern zu. Die Anzahl der Isolate pro Cluster reichte von einem bis zu 64 Stämmen. Dabei umfassten drei Cluster zwei Drittel der 155 typisierten Stämme (66.5\%). Zur Speziesidentifizierung wurde von jeweils einem oder zwei Stämmen aus 12 verschiedenen Clustern mit insgesamt 144 Isolaten eine 16S rDNA Sequenzierung durchgeführt. Tabelle 3 zeigt die dabei erhaltene Zuordnung der Restriktionsprofile zu 10 verschiedenen CNS-Spezies. In den drei dominierenden Clustern wurden $S$. saprophyticus, S. equorum und S. lentus nachgewiesen. Daher scheinen bei den CNS aus Mastitismilchproben von Schafen und Ziegen in der Schweiz bestimmte Spezies $\mathrm{zu}$ dominieren, wenn auch viele verschiedene als Mastitiserreger bei Kleinwiederkäuern verbreitet sind.

In anderen Studien (Rodrigues da Silva et al., 2004; Contreras et al., 2007; Marogna et al., 2010) wurde ebenfalls eine Vielzahl von CNS-Spezies aus Milchproben von Schafen und Ziegen isoliert. Trotz geographischer Unterschiede und Unterschieden zwischen den Tierarten dominierten dabei oft S. epidermidis, S. caprae, S. simulans, S. 
chromogenes und S. xylosus. Überdies scheint ein Zusammenhang sowohl zwischen der Zellzahl und der CNS-Spezies wie auch zwischen dem Vorkommen von Antibiotikaresistenzen und der CNS-Spezies zu bestehen (Rodrigues da Silva et al. 2004; Schaeren und Haldemann, 2009).

\section{Antibiotikaresistenz}

Die Ergebnisse der Empfindlichkeitsprüfung von $S$. aureus und CNS aus Mastitismilchproben von Kleinwiederkäuern sind in den Tabellen 1 und 2 aufgeführt. Bislang lagen nur wenige aktuelle Daten zur Resistenzsituation von Mastitiserregern bei Schafen und Ziegen vor und für die Schweiz fehlten solche bis anhin ganz. Zudem wird ein Vergleich der Ergebnisse durch variierende Rahmenbedingungen wie der Auswahl der Mikroorganismen und Antibiotika oder den Verfahren und Kriterien zur Resistenzbeurteilung erschwert (Schwarz et al., 2010).

Bei den 67 aus Mastitismilchproben von Kleinwiederkäuern isolierten S. aureusStämmen wurden Resistenzen gegen Ampicillin, Erythromycin, Penicillin G und Tetracyclin gefunden (Tab. 1). Knapp ein Drittel der S. aureus-Stämme wies jeweils eine Penicillinresistenz (31.3\%) und eine Ampicillinresistenz (29.9\%) auf. Diese lagen in der Regel in Kombination vor. Zudem war jeweils ein Stamm resistent gegen die Kombination von Penicillin G und Erythromycin oder Penicillin G und Tetracyclin. Mit Ausnahme der Penicillin- und Ampicillinresistenzen verfügten die 67 isolierten S. aureus-Stämme jedoch nur selten über Resistenzen gegen die getesteten Antibiotika. In einer brasilianischen Studie (Rodrigues da Silva et al., 2004) erwiesen sich ebenfalls etwa ein Drittel der aus Ziegenmilchproben isolierten S. aureusStämme $(36 \%)$ als Penicillin-resistent, während in Studien aus Italien (Moroni et al., 2005b; Lollai et al. 2008), Norwegen (Mørk et al., 2005) oder Slowenien (Pengov und Ceru, 2003) nur vereinzelte $S$. aureus-Stämme aus Schaf- oder Ziegenmilch eine solche Resistenz aufwiesen.

Bei den 208 aus Mastitismilchproben von Kleinwiederkäuern isolierten CNSStämmen wurden Resistenzen gegen Ampicillin, Erythromycin, Oxacillin, Penicillin 
G, Sulfamethoxazol/Trimethoprim und Tetracyclin gefunden (Tab. 2). Dabei dominierten Resistenzen gegen Erythromycin (10.6\%) und Penicillin (8.2\%). CNS waren dabei im Vergleich zu den untersuchten S. aureus-Stämmen signifikant weniger häufig resistent gegen Penicillin und Ampicillin sowie signifikant häufiger resistent gegen Erythromycin ( $\mathrm{P}<0.001$; Chi-Quadrat Test). Auch erwiesen sich $4.8 \%$ der isolierten CNS-Stämme als resistent gegen Oxacillin, während eine solche Resistenz bei den untersuchten $S$. aureus-Stämmen nicht gefunden wurde. In anderen Studien (Rodrigues da Silva et al., 2004; Lollai et al., 2008) wurden bei aus Milchproben von Schafen und Ziegen isolierten CNS-Stämmen oft höhere Resistenzraten gegen Penicillin, insbesondere höhere als bei $S$. aureus, gefunden. Auch erwiesen sich in der Studie von Rodrigues da Silva et al. (2004) 22.5\% der aus Ziegenmilch isolierten CNS als Oxacillin-resistent. Zudem zeigten diese Autoren, dass zwischen verschiedenen CNS-Spezies Unterschiede in den Resistenzprofilen und -raten vorlagen (Rodrigues da Silva et al., 2004).

Für die Schweiz lagen bis anhin lediglich Resistenzdaten $\mathrm{zu}$ bovinen Mastitiserregern vor. Bei bovinen S. aureus wurden dabei insbesondere Penicillinund Ampicillinresistenzen gefunden, wobei die Häufigkeit in der Regel bei $\leq 15 \%$ lag (Busato et al., 2000; Corti et al.; 2003; Roesch et al., 2006). Im Gegensatz zu den vorliegenden Ergebnissen von Kleinwiederkäuern wurden bei bovinen CNS oft höhere Resistenzraten gegen Penicillin (>30\%) sowie Ampicillin nachgewiesen (Corti et al., 2003; Roesch et al. 2006). So wiesen in der Studie von Corti et al. (2003) von den bovinen S. aureus-Stämmen 9\% eine Penicillin- und 7\% eine Ampicillinresistenz auf, während 31\% der CNS gegen Penicillin und 26\% gegen Ampicillin resistent waren. 


\section{Schlussfolgerungen}

Koagulase-negative Staphylokokken (CNS) dominieren als Erreger von Mastitiden bei Schafen und Ziegen in der Schweiz. Bei gehäuftem Auftreten von Mastitiden sollte auch bei Kleinwiederkäuern eine bakteriologische Abklärung der Ursache durchgeführt werden, denn insbesondere bei Ziegenmilch sind Zellzahlen zur Erkennung von Mastitiden nur beschränkt tauglich (Schaeren et al., 2007). Insgesamt dominierten bestimmte CNS-Spezies, wenn auch viele verschiedene CNS als Mastitiserreger bei Kleinwiederkäuern nachgewiesen wurden. Dabei könnten sich verschiedene CNS-Spezies in ihrer Pathogenität und auch hinsichtlich des Vorkommens von Antibiotikaresistenzen unterscheiden. Insbesondere in Betrieben mit Bestandesproblemen kann es daher angezeigt sein, Infektionen mit CNS genauer zu untersuchen. Die festgestellten Unterschiede in der Empfindlichkeit der Mastitiserreger gegenüber den getesteten Antibiotika unterstreichen die Bedeutung der Resistenzprüfung, zusammen mit einer zuverlässigen Identifizierung der Erreger. Allerdings sind die Aussagen unter Praxisbedingungen zu relativieren, da die Prüfung in vitro erfolgte. Die Ergebnisse der vorliegenden Arbeit stellen eine erste Bestandesaufnahme zur Resistenzsituation bei $S$. aureus und CNS von Schafen und Ziegen in der Schweiz dar. 


\section{Literatur}

Bergonier D., de Crémoux R., Rupp R., Lagriffoul G., Berthelot X.: Mastitis of dairy small ruminants. Vet. Res. 2003, 34: 689-716.

Busato A., Trachsel P., Schällibaum M., Blum J.W.: Udder health and risk factors for subclinical mastitis in organic dairy farms in Switzerland. Prev. Vet. Med. 2000, 44: 205-220.

Clinical and Laboratory Standards Institute, 2008. Performance standards for antimicrobial susceptibility testing; sixteenth informational supplement M100-S18, vol. 28. Clinical and Laboratory Standards Institute, Wayne, PA.

Clinical Laboratory Standards Institute, 2010: Performance standards for antimicrobial susceptibility testing; twentieth informational supplement M100-S20, Vol. 30. Clinical and Laboratory Standards Institute, Wayne, PA.

Contreras A., Sierra D., Sánchez A., Corrales J.C., Marco J.C., Paape M.J., Gonzalo C.: Mastitis in small ruminants. Small Rumin. Res. 2007, 68: 145-153.

Corti S., Sicher D., Regli W., Stephan R.: Aktuelle Daten zur Antibiotikaresistenz der wichtigsten bovinen Mastitiserreger in der Schweiz. Schweiz. Arch. Tierheilkd. 2003, 145: 571-575.

Fantelli K., Stephan R.: Validierung der DNAse-reaktion zur Identifizierung von Staphylococus aureus Stämmen im Rahmen der Mastitisdiagnostik. Schweiz Arch. Tierheilkd. 2003, 145: 76-79.

Gonzalo C., Ariznabarreta A., Carriedo J.A., San Primitivo F.: Mammary pathogens and their relationship to somatic cell count and milk yield losses in dairy ewes. J. Dairy Sci. 2002, 85: 1460-1467.

Halasa T., Huijps K., Østerås O., Hogeveen H.: Economic effects of bovine mastitis and mastitis management: A review. Vet. Q. 2007, 29: 18-31.

Juretschko S., Loy A., Lehner A., Wagner M.: The microbial community composition of a nitrifying-denitrifying activated sludge from an industrial sewage treatment plant analyzed by the full-cycle rRNA approach. Syst. Appl. Microbiol. 2002, 25: 8499. 
Lollai S.A., Ziccheddu M., Di Mauro C., Manunta D., Nudda A., Leori G.: Profile and evolution of antimicrobial resistance of ovine mastitis pathogens (1995-2004). Small Rumin. Res. 2008, 74: 249-254.

Marogna G., Rolesu S., Lollai S., Tola S., Leori G.: Clinical findings in sheep farms affected by recurrent bacterial mastitis. Small Rumin. Res. 2010, 88: 119-125.

Maurer J., Schaeren W.: Eutergesundheit und Zellzahlen bei Milchschafen. Agrarforschung 2007, 14: 162-167.

McDougall S., Pankey W., Delaney C., Barlow J., Murdough P.A., Scruton D.: Prevalence and incidence of subclinical mastitis in goats and dairy ewes in Vermont, USA. Small Rumin. Res. 2002, 46: 115-121.

Mørk T., Tollersrud T., Kvitle B., Jørgensen H.J., Waage S.: Comparison of Staphylococcus aureus genotypes recovered from cases of bovine, ovine, and caprine mastitis. J. Clin. Microbiol. 2005, 43: 3979-3984.

Moroni P., Pisoni G., Antonini M., Ruffo G., Carli S., Varisco G., Boettcher P.: Subclinical mastitis and antimicrobial susceptibility of Staphylococcus caprae and Staphylococcus epidermidis isolated from two Italian goat herds. J. Dairy Sci. 2005a, 88: 1694-1704.

Moroni P., Pisoni G., Vimercati C., Rinaldi M., Castiglioni B., Cremonesi P., Boettcher P.: Characterization of Staphylococcus aureus isolated from chronically infected dairy goats. J. Dairy Sci. 2005b, 88: 3500-3509.

Pengov A., Ceru S.: Antimicrobial drug susceptibility of Staphylococcus aureus strains isolated from bovine and ovine mammary glands. J. Dairy Sci. 2003, 86: 3157-3163.

Pyörälä S., Taponen S.: Coagulase-negative staphylococci - Emerging mastitis pathogens. Vet. Microbiol. 2009, 134: 3-8.

Rodrigues da Silva E., Pimenta Siqueira A., Dias Martins J.C., Barbosa Ferreira W.P., da Silva N.: Identification and in vitro antimicrobial susceptibility of Staphylococcus species isolated from goat mastitis in the Northeast of Brazil. Small Rumin. Res. 2004, 55: 45-49.

Roesch M., Perreten V., Doherr M.G., Schaeren W., Schällibaum M., Blum J.W.: Comparison of antibiotic resistance of udder pathogens in dairy cows kept on organic and on conventional farms. J. Dairy Sci. 2006, 89: 989-997. 
Santos O.C., Barros E.M., Brito M.A., Bastos Mdo C., Dos Santos K.R., GiambiagiDemarval M.: Identification of coagulase-negative staphylococci from bovine mastitis using RFLP-PCR of the groEL gene. Vet. Microbiol. 2008, 130: 134-140.

Schaeren W., Maurer J.: Häufigkeiten subklinischer Euterinfektionen und individuelle Zellzahlen in drei Ziegenherden im Verlaufe einer gesamten Laktation. Schweiz. Arch. Tierheilkd. 2006, 148: 641-648.

Schaeren W., Jakob E., Maurer J., Ryffel S.: Ziegen- und Schafmilchproduktion Qualität zahlt sich aus: Merkblatt für die Praxis. ALP aktuell 2007, 29: 1-4.

Schaeren W., Haldemann C.: Vorkommen verschiedener Arten von Staphylokokken als Euterinfektionserreger bei Ziegen. Forum Kleinwiederkäuer 2009, 12: 14-17.

Schwarz S., Silley P., Simjee S., Woodford N., van Duijkeren E., Johnson A.P., Gaastra W.: Assessing the antimicrobial susceptibility of bacteria obtained from animals. Vet. Microbiol. 2010, 141: 1-4.

Zadoks R.N., Watts J.L.: Species identification of coagulase-negative staphylococci: Genotyping is superior to phenotyping. Vet. Microbiol. 2009, 134: 20-28.

Zweifel C., Muehlherr J.E., Ring M., Stephan R.: Influence of different factors in milk production on standard plate count of raw small ruminant's bulk-tank milk in Switzerland. Small Rumin. Res. 2005, 58: 63-70. 


\section{Tabellen}

Tabelle 1: Minimale Hemmkonzentrationen (MHK) bei den 67 aus Mastitismilchproben von Schafen und Ziegen isolierten Staphylococcus aureus-Stämmen.

\begin{tabular}{|c|c|c|c|c|c|c|c|c|c|c|c|}
\hline \multirow{2}{*}{ Wirkstoff } & \multicolumn{10}{|c|}{ Anzahl Stämme mit MHK $(\mu \mathrm{g} / \mathrm{ml})$ von } & \multirow{2}{*}{$\begin{array}{l}\text { Anteil } \\
\text { resistenter } \\
\text { Stämme }\end{array}$} \\
\hline & $<0.125$ & 0.125 & 0.25 & 0.5 & 1 & 2 & 4 & 8 & 16 & 32 & \\
\hline Amoxicillin/Clavulansäure ${ }^{\mathrm{a})}$ & & & 58 & 5 & & 2 & 2 & & & & $0 \%$ \\
\hline Ampicillin & 41 & 5 & 1 & 14 & 6 & & & & & & $29.9 \%$ \\
\hline Cefazolin & & & & & & & 67 & & & & $0 \%$ \\
\hline Cephalotin & & & & & 67 & & & & & & $0 \%$ \\
\hline Enrofloxacin & 47 & 18 & 1 & 1 & & & & & & & $0 \%$ \\
\hline Erythromycin & 2 & 20 & 42 & 2 & & & & 1 & & & $1.5 \%$ \\
\hline Gentamicin & & & & 67 & & & & & & & $0 \%$ \\
\hline Oxacillin & & 63 & 4 & & & & & & & & $0 \%$ \\
\hline Penicillin G & 45 & 1 & 5 & 7 & 5 & 1 & 3 & & & & $31.3 \%$ \\
\hline Sulfamethoxazol/ Trimethoprim ${ }^{\text {a) }}$ & & & 67 & & & & & & & & $0 \%$ \\
\hline Tetracyclin & & & & 61 & 1 & 1 & & 2 & 2 & & $3.0 \%$ \\
\hline
\end{tabular}

Die für jedes Antibiotikum getesteten Konzentrationsbereiche sind weiss hinterlegt (Sensititre custom plate). Werte über oder unter diesen Bereichen stehen für MHK-Werte grösser als die höchste getestete Konzentration bzw. kleiner oder gleich als die geringste getestete Konzentration. Die vertikalen schwarzen Balken zeigen die jeweiligen, im CLSI Supplement M100-S18 für S. aureus aufgeführten Breakpoints (Clinical and Laboratory Standards Institute, 2008).

a) Konzentrationen für Amoxicillin (Verhältnis Amoxicillin/Clavulansäure 2:1) und Trimethoprim (Verhältnis Sulfamethoxazol/ Trimethoprim 19:1) 
Tabelle 2: Minimale Hemmkonzentrationen (MHK) bei den 208 aus Mastitismilchproben von Schafen und Ziegen isolierten Koagulase-negativen Staphylokokken-Stämmen.

\begin{tabular}{|c|c|c|c|c|c|c|c|c|c|c|c|}
\hline \multirow{2}{*}{ Wirkstoff } & \multicolumn{10}{|c|}{ Anzahl Stämme mit MHK $(\mu \mathrm{g} / \mathrm{ml})$ von } & \multirow{2}{*}{$\begin{array}{c}\text { Anteil } \\
\text { resistenter } \\
\text { Stämme }\end{array}$} \\
\hline & $<0.125$ & 0.125 & 0.25 & 0.5 & 1 & 2 & 4 & 8 & 16 & 32 & \\
\hline Amoxicillin/Clavulansäure ${ }^{a}$ & & & 205 & 3 & & & & & & & $0 \%$ \\
\hline Ampicillin & 182 & 20 & 4 & & 1 & 1 & & & & & $1.0 \%$ \\
\hline Cefazolin & & & & & & & 208 & & & & $0 \%$ \\
\hline Cephalotin & & & & & 207 & & & 1 & & & $0 \%$ \\
\hline Enrofloxacin & 87 & 100 & 15 & 3 & 2 & 1 & & & & & $0 \%$ \\
\hline Erythromycin & 20 & 61 & 74 & 9 & 4 & 12 & 6 & 22 & & & $10.6 \%$ \\
\hline Gentamicin & & & & 207 & & 1 & & & & & $0 \%$ \\
\hline Oxacillin & & 125 & 73 & 8 & 1 & 1 & & & & & $4.8 \%$ \\
\hline Penicillin G & 191 & & 8 & 4 & 2 & & 1 & 1 & 1 & & $8.2 \%$ \\
\hline Sulfamethoxazol/ Trimethoprim ${ }^{\text {a) }}$ & & & 205 & 2 & & & 1 & & & & $0.05 \%$ \\
\hline Tetracyclin & & & & 188 & 3 & & 1 & & 16 & & $7.7 \%$ \\
\hline
\end{tabular}

Die für jedes Antibiotikum getesteten Konzentrationsbereiche sind weiss hinterlegt (Sensititre custom plate). Werte über oder unter diesen Bereichen stehen für MHK-Werte grösser als höchste getestete Konzentration bzw. kleiner oder gleich als die geringste getestete Konzentration. Die vertikalen schwarzen Linien zeigen die jeweiligen, im CLSI Supplement M100-S20 für Koagulase-negative Staphylokokken aufgeführten Breakpoints (Clinical and Laboratory Standards Institute, 2010).

a) Konzentrationen für Amoxicillin (Verhältnis Amoxicillin/Clavulansäure 2:1) und Trimethoprim (Verhältnis Sulfamethoxazol/ Trimethoprim 19:1) 
Tabelle 3: Charakterisierung von 144 aus Schaf- und Ziegenmilch isolierten Koagulase-negativen Staphylokokken-Stämmen.

\begin{tabular}{cccl}
\hline $\begin{array}{c}\text { Anzahl CNS-Stämme } \\
\text { (Schaf-/Ziegenmilch) }\end{array}$ & $\begin{array}{c}\text { PCR-RFLP- } \\
\text { Profil }^{\text {a) }}\end{array}$ & Speziesidentifikation (16S rDNA) \\
\hline 64 & $(36 / 28)$ & $\mathrm{A}$ & Staphylococcus saprophyticus \\
27 & $(9 / 18)$ & $\mathrm{B}$ & Staphylococcus equorum \\
12 & $(9 / 3)$ & $\mathrm{C}$ & Staphylococcus lentus \\
9 & $(5 / 4)$ & $\mathrm{D}$ & Staphylococcus epidermidis \\
7 & $(4 / 3)$ & $\mathrm{E}$ & Staphylococcus chromogenes \\
6 & $(4 / 2)$ & $\mathrm{F}$ & Staphylococcus capitis \\
6 & $(2 / 4)$ & $\mathrm{G}$ & Staphylococcus succinus \\
4 & $(3 / 1)$ & $\mathrm{H}$ & Staphylococcus xylosus \\
3 & $(2 / 1)$ & $\mathrm{I}$ & Staphylococcus haemolyticus \\
2 & $(2 / 0)$ & $\mathrm{J}$ & Staphylococcus simulans \\
2 & $(1 / 1)$ & $\mathrm{K}$ & Staphylococcus succinus \\
2 & $(1 / 1)$ & $\mathrm{L}$ & Staphylococcus epidermidis \\
\hline
\end{tabular}

a) Restriktionsprofil-Analyse (PCR-RFLP) des groEL-Gens (Santos et al., 2008). 


\section{Danksagung}

An dieser Stelle möchte ich mich bei all denjenigen bedanken, die zum Gelingen dieser Arbeit beigetragen haben.

Besonderer Dank geht an:

Herrn Prof. Dr. Roger Stephan, Institut für Lebensmittelsicherheit und -hygiene, Vetsuisse-Fakultät Universität Zürich, für die Möglichkeit zur Erstellung dieser Dissertation.

Herrn Prof. Michael Hässig für die Uebernahme des Korreferates.

Herr PD Dr. Claudio Zweifel, Institut für Lebensmittelsicherheit und -hygiene, Vetuisse-Fakultät, für die tatkräftige Hilfe bei der Erstellung des Manuskriptes.

Dem gesamtem ILS Team, insbesondere Dr. Sabrina Corti und Nicole Giezendanner für die gute und geschätzte Unterstützung im Labor. 


\section{Lebenslauf}

Name, Vorname:

Geburtsatum:

Geburtsort:

Nationalität:

Heimatort:

08.1992-06.1999

24.06.1999

09.1999-09.2005

30.09.2005

02.2008-05.2010

10.2005-02.2006

03.2006-03.2010

Seit 04.2010
Kunz, Franziska

06.04.1979

Ruswil

Schweiz

Ruswil und Grosswangen

Kantonsschule Sursee und Willisau

Matura Kantonsschule Willisau

Studium Veterinärmedizin, Vetsuisse Fakultät Universität Bern

Abschlussprüfung vet. med., Vetsuisse-Fakultät, Universität Bern

Anfertigung der Dissertation

Praktikum Nutztierpraxis, Dr. med. vet. D. Hotz, Baar

Assistentin Nutztierpraxis Casura\&Partner, Oberentfelden

Assistentin in den beiden Gemischtpraxen Dr. med. vet. M. Stöckli, Rothenburg und Dr. med. vet. Urs Wolfisberg, Neuenkrich 